\title{
Position statement on cancer nursing's potential to reduce the growing burden of cancer across the world
}

\author{
Patsy Yates ${ }^{1,2}$ - Andreas Charalambous ${ }^{3,4}$ - Laura Fennimore ${ }^{5}$ - Brenda Nevidjon ${ }^{5}$ - Winnie K. W. So ${ }^{6,7}$. \\ Eunyoung E. Suh ${ }^{6,8} \cdot$ Emma Woodford $^{3}$. Annie Young 9,10 \\ Published online: 12 March 2021 \\ (C) Asian Oncology Nursing Society, European Oncology Nursing Society, International Society of Nurses in Cancer Care, Multinational Association of \\ Supportive Care in Cancer, Oncology Nursing Society. All rights reserved 2021
}

\section{The global burden of cancer is growing}

Cancer is the first or second leading cause of premature death (i.e. at ages 30-69 years) in 134 of 183 countries, and it ranks third or fourth in an additional 45 countries $^{1}$. An estimated 18.1 million new cancer cases and 9.6 million cancer-related deaths occurred worldwide in $2018^{2}$. The incidence of cancer globally is expected to exceed 27 million new cancer cases per year by 2040 in part due to the ageing of the population and greater susceptibility to ageing-related diseases such as cancer ${ }^{3}$. This estimated increase in cancer incidence will occur in all countries, but the predicted increase will be proportionately greatest in low and medium countries $^{3}$.Cancer is also a disease associated with significant morbidity. It is estimated that the total annual economic cost of cancer in 2010 was US $\$ 1.16$ trillion per year ${ }^{4}$.

There is extensive evidence which indicates that the burden of cancer can be substantially reduced. Approximately 30 $50 \%$ of cancers can be prevented, early diagnosis and access to evidence-based treatments can significantly improve survival, and evidence-based supportive care interventions can significantly improve safety, reduce morbidity, optimise

This position statement is being published simultaneously in Asia-Pacific Journal of Oncology Nursing, Cancer Nursing, European Journal of Oncology Nursing, Oncology Nursing Forum and Supportive Care in Cancer.

Patsy Yates

p.yates@qut.edu.au

1 International Society of Nurses in Cancer Care, Vancouver, Canada

2 Faculty of Health, Queensland University of Technology, O Block, D Wing, Level 7, Kelvin Grove, Brisbane, Queensland, Australia

3 European Oncology Nursing Society, Brussels, Belgium

4 Cyprus University of Technology, Limassol, Cyprus

5 Oncology Nursing Society, Pittsburg, PA, USA quality of life, and improve the experiences of health care for the person affected by cancer and their family. Reducing the burden of cancer is not only about limiting the numbers of those affected by cancer. It also requires actions to improve the person's experience of cancer across the disease trajectory.

Cancer control has been a growing priority of governments globally and the World Health Organization (WHO). The 2017 World Health Assembly resolution on cancer prevention and control proposed an integrated approach to cancer control from a public policy perspective. The guiding principle of these WHO efforts is that health is a basic human right, and in order to respect that right, health services need to be provided through a universal health coverage system that leaves no one behind ${ }^{5}$.

\section{The nursing workforce is essential to cancer control}

There are over 20 million nurses and midwives worldwide making them the largest group of health professionals who

6 Asian Oncology Nursing Society, Seoul, Republic of Korea

7 The Nethersole School of Nursing, The Chinese University of Hong Kong, Ma Liu Shui, Hong Kong

8 College of Nursing, Research Institute of Nursing Science, Seoul National University, Seoul, South Korea

9 Multinational Association of Supportive Care in Cancer, Aurora, Canada

10 University of Warwick, Coventry, England 
are well placed to reduce the burden of cancer. In many countries, nurses are at the frontline to reduce the burden of cancer as they provide essential primary health care services which includes education focused on prevention and early detection of cancer. Nurses also reduce the burden of cancer by delivering complex treatments, ensuring timely and safe minimization of complications, providing care for individuals and their families across a range of care settings, and empowering individuals and their families to assume self-management of the disease ${ }^{6,7}$. Achieving the goal of universal health coverage to reduce the burden of cancer therefore urgently requires that nursing services are not only strengthened, but that they are optimised and extended.

\section{Nurses reduce the burden of cancer across the cancer trajectory}

Nurses contribute to reducing the burden of cancer across the entire pathway (see Table 1).

Cancer nurses have distinct functions which are critical to the success of modern cancer care, especially in this time of unprecedented change to the nature of cancer treatment and control. These broad impacts of nursing services in cancer control are demonstrated in a growing body of evidence from studies in high resource countries. One recent scoping review of 214 studies of interventions led or delivered by cancer nurses involving 247,550 participants concluded that nursing interventions were delivered across the cancer continuum from prevention and risk reduction to survivorship. The interventions included case management, surveillance, teaching, counselling, and guidance, and a variety of treatments and procedures. Most of the interventions were delivered by a specialist or advanced practice nurses, highlighting the need for a robust career structure and education for nurses ${ }^{6}$.

\section{An opportunity to realise the essential role of nurses}

There is substantial unrealised potential for nurses to reduce the burden of cancer across the world. In low- and middleincome countries, there is an opportunity for government and non-government organizations to improve the working conditions of nurses, such as low pay, workforce shortages, and a lack of opportunities for professional growth ${ }^{7,8}$. In many high resource countries, while specialised cancer nursing roles have developed over many decades and there is a strong evidence base to support them, there is substantial variation in access to such services and regulatory, system level and professional barriers which limit service delivery.
Table 1 Examples of the Contribution of Nurses across the Cancer Trajectory $^{7}$

- Cancer education:

- increase adherence to screening guidelines

- counsel patients on access to local health systems to decrease delay in care

- educate community health workers about cancer to raise awareness and appropriately refer a patient for further evaluation

- Prevention:

- implement preventive interventions at both the individual and the community level given their accessibility to and active role in the community

- identify risk factors, and communicate and teach individuals, families, and communities to change behaviors to reduce risk factors (e.g. smoking cessation; uptake of vaccinations; inactivity; poor diet; sun exposure; alcohol intake)

- Screening and early detection:

- perform the broad range of interventions that contribute to screening, early detection, and even treatment of precancerous lesions

- Treatment:

- clinically assess and educate patients and family members about treatments by addressing patient fears and providing information about potential adverse effects

- identify adverse effects early and so that steps can be taken to avoid complications, avoid treatment delays, and ensure safety

- conduct a comprehensive assessment of the health and supportive care needs of patients with cancer

- educate and provide psychosocial and spiritual support by sharing and apply knowledge of cancer and treatment modalities and adverse effects.

- Palliative care:

- assess, identify, and manage not only pain but also the physical, psychosocial, spiritual, and cultural needs of patients and their families throughout the cancer journey and at the end of life

- Survivorship:

- psychosocial support and healthy lifestyle promotion to improve quality of life and behavioral outcomes for patient

- assess and educate about physical changes and late effects following cancer treatment

- Research:

- develop new knowledge

- collaborate with epidemiologists, public health and researchers from other disciplinary backgrounds

\section{Call to action}

It is our position that:

- Nurses are essential to the success of reducing cancer incidence, improving survival and quality of life, and providing better palliative care ${ }^{7}$.

- Well-prepared cancer nurses have demonstrated wideranging impacts across the spectrum of cancer care in many high-income countries. To benefit from this expertise, substantial efforts to build nursing workforce capacity are required in low- and middle-income and high-income 
countries where the nursing workforce does not have access to adequate education ${ }^{7}$.

- The role of the cancer nurse needs to be formally recognised as a key part of global cancer control efforts and appropriate nursing resources should be mandated within all cancer control programs ${ }^{7}$.

- Government and non-government organisations must optimise the substantial resource of nurses to ensure that the burden of cancer is reduced worldwide. This requires urgent action to ensure:

- Adequate education for nurses

- Removal of regulations which act as barriers to nurses' practice

- Safe staffing levels

- Safe work environments

- Investment in innovative nurse-led models and practices

- Greater nursing leadership in cancer control

- Stronger partnerships between all involved in cancer control

- Research to advance the evidence base for cancer nursing

Code availability Not applicable.

Data availability Not applicable.

\section{Declarations}

Ethics approval Not applicable.

Consent for publication Not applicable.

Consent to participate Not applicable.

Conflict of interest The authors declare no conflict of interests.

\section{References}

1. Cao B, Soerjomataram I, Bray F (2020) The burden and prevention of premature deaths from noncommunicable diseases, including cancer: a global perspective. In: Wild CP, Weiderpass E, Stewart BW (eds) World Cancer Report: Cancer Research for Cancer Prevention. International Agency for Research on Cancer, Lyon Available from: http://publications.iarc.fr/586. Licence: CC BYNC-ND 3.0 IGO. Accessed 7 March 2021

2. Bray F, Ferlay J, Soerjomataram I, Siegel RL, Torre LA, Jemal A (2018) Global cancer statistics 2018: GLOBOCAN estimates of incidence and mortality worldwide for 36 cancers in 185 countries. CA Cancer J Clin. 68(6):394-424

3. Fidler-Benaoudia M, Bray F (2020) Transitions in human development and the global cancer burden. In: Wild CP, Weiderpass E, Stewart BW (eds) World Cancer Report: Cancer Research for Cancer Prevention. International Agency for Research on Cancer, Lyon Available from: http://publications.iarc.fr/586. Licence: CC BY-NC-ND 3.0 IGO. Accessed 7 March 2021

4. Seventieth World Health Assembly (2017) Resolution WHA70.12. Cancer prevention and control in the context of an integrated approach. World Health Organization, Geneva Available from: https://apps.who.int/iris/handle/10665/275676. Accessed 7 March 2021

5. Prager GW, Braga S, Bystricky B, Qvortrup C, Criscitiello C, Esin E, Sonke GS, Martínez GA, Frenel JS, Karamouzis M, Strijbos M, Yazici O, Bossi P, Banerjee S, Troiani T, Eniu A, Ciardiello F, Tabernero J, Zielinski CC, Casali PG, Cardoso F, Douillard JY, Jezdic S, McGregor K, Bricalli G, Vyas M, Ilbawi A (2018) Global cancer control: responding to the growing burden, rising costs and inequalities in access. ESMO Open 3:e00285. https://oi.org/ 10.1136/esmoopen-2017-000285)

6. Charalambous A, Wells M, Campbell P, Torrens C, Östlund U, Oldenmenger W, Patiraki E, Sharp L, Nohavova I, DomenechCliment N, Eicher M, Farrell C, Larsson M, Olsson C, Simpson M, Wiseman T, Kelly D (2018) A scoping review of trials of interventions led or delivered by cancer nurses. International Journal of Nursing Studies 86:36-43

7. Challinor JM, Galassi AL, Al-Ruzzieh MA, Bigirimana JB, Buswell L, So WKW, Steinberg AB, Williams MJ (2016) Nursing's potential to address the growing cancer burden in low- and middle-income countries. Journal of Global Oncology 2(3):154-163

8. So WKW, Cumming GG, Ayala de Calvo LE, Day SW, Houlahan K, Nevidjon BM, Schneider J, Teahon T, Were PA (2016) Enhancement of oncology nursing education in low- and middleincome countries: challenges and strategies. Journal of Cancer Policy 8:10-16

Publisher's note Springer Nature remains neutral with regard to jurisdictional claims in published maps and institutional affiliations. 